\title{
基于 STEM 的跨学科学习教学设计案例研究
}

\section{A case study of STEM-based interdisciplinary learning teaching design}

\author{
杨洁 \\ Jie Yang \\ 首都师范大学附属中学大兴南校区 中国·北京 102600
}

Daxing South Campus, High School Affiliated to Capital Normal University, Beijing 102600, China

\begin{abstract}
摘 要: 作为一种跨学科式教育, STEM 教育逐渐受到国际科学教育的关注和重视, 成为各国科学教育的一门新兴课程。 该研究提出了基于 STEM 教育理念的 $5 \mathrm{E}$ 探究式教学模式, 强调学习者以主题项目为切入点展开学习, 激发学习兴趣, 通 过迭代设计制作出解决实际问题的产品, 促进学习者问题解决能力、科学探究能力的提升。该研究设计并实施的 “暴躁的 天气” 系列棵程，通过以首都师范大学附属中学大兴南校区五年级学生为研究对象进行的单组前后测实验中发现：基于 STEM 的跨学科教学能够激发学生的学习兴趣, 且有效促进学生科学探究能力的发展。
\end{abstract}

Abstract: As a kind of interdisciplinary education, STEM education has gradually received the attention and attention of international science education and has become an emerging course in science education in various countries. The research puts forward a $5 \mathrm{E}$ inquiry-based teaching model based on the concept of STEM education, emphasizing that learners start learning with thematic projects as the entry point, stimulate learning interest, and produce products that solve practical problems through iterative design, and promote learners' problem-solving skills and science Enhancement of inquiry ability. The research designed and implemented a series of courses on "Crumpy Weather" . Through a single-group pre-test experiment conducted with fifth-grade students on the Daxing South Campus of the High School Affiliated to Capital Normal University, it was found that STEM-based interdisciplinary teaching can stimulate students' Learning interest, and effectively promote the development of students' scientific inquiry ability.

关键词: STEM 教育; 跨学科学习; 科学探究能力;

Keywords: STEM education; interdisciplinary learning; scientific inquiry ability;

基金项目: 北京市教育科学 “十三五” 规划一般棵题 “基于 STEM 理念的跨学科教学对小学生科学素养的培养研究”, 课题编号: CDDB19285

DOI: $10.36012 /$ sde.v2i12.2597

2017 年 6 月《中国 STEM 教育白皮书》指出，应将跨 学科 STEM ( 科学、技术、工程、艺术与数学) 教育纳入国 家创新型人才培养战略，是全社会共同参与的教育创新实 践。2 017 年 2 月，教育部颁布了《全日制义务教育小学科 学课程标准》( 以下简称 “标准” ), 我国首次以科学课为依托, 提出 STEM 教育的标准。该标准提出, 科学教学应该引导 学生通过探究活动, 获得科学基础知识和技能, 倡导通过跨 学科方式学习, 即融合科学、技术、工程、数学为一体的
STEM 教学, 以一种项目学习、问题解决为导向的课程组织 方式运用于科学教学中。这是我国首次以科学课为依托, 提 出 STEM 教育的标准。可见, 跨学科 STEM 教学在当今教 育领域有着非常重要的地位。那么, 如何运用 STEM 跨学 科知识融合的理念，培养学生综合实践能力、问题解决能力 和创新能力，成为教育工作者思考的问题。鉴于此，笔者将 如何运用 STEM 跨学科理念提升小学生科学探究能力作为 本研究的重点, 将 STEM 教育从理念层面落实到实践层面,

【作者简介】杨洁，(1986)，女，山西阳泉人，中学二级教师，从事教学方面研究。 
并对其教学效果进行分析, 以期为 STEM 教育的教学实践 研究与发展提供更多的参考。

\section{1 基于 STEM 理念的 $5 E$ 探究式教学模式}

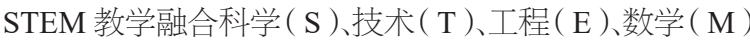
等多学科 ( STEM) 内容, 实现跨学科知识之间的整合。 STEM 跨学科整合的最重要工作就是将学习者真实的生活、 学习情境融人到主题项目或主题问题的设计当中。本研究在 构建基于 STEM 理念的课程的教学设计模式时, 借鉴了贝 比 (R.Bybee ) 发展起来的建构主义 $5 \mathrm{E}$ 教学模式, 融合美国《下 一代科学标准》NGSS 提出的科学与工程实践理念, 结合自 己在首都师范大学附属中学大兴南校区指导 STEM 课程开 发的经验, 建构了以 “探究” 为核心的基于 STEM 理念 $5 \mathrm{E}$ 教学模式, 包含了参与 (Engage) 、探究 (Explore)、解释 (Explain)、拓展（Elaborate）和评价（Evaluate）五个环节, 针对主题项目内容, 各个环节循环交替进行, 其中 “探究”
既是 $5 \mathrm{E}$ 教学活动的中心环节, 也是贯穿于整个 STEM 教学 过程的核心思想。当一个探究节点结束之后, 又会在此基础 上以该结束点为起点, 触发新的节点和新一轮的探究过程。 合理的进行主题内容设计, 将知识情景化、生活化, 学习者 探究性地参与学习实践, 学会自主运用学科知识, 创造性解 决问题。

\section{2 基于 STEM 理念的课程《暴躁的天气》 教学设计}

\section{1 基于 STEM 理念的课程《暴躁的天气》教学 目标分析}

基于 STEM 理念的课程《暴躁的天气》融合了 $\mathrm{S}$ (科学)、 $\mathrm{T}$ (技术)、 $\mathrm{E}$ ( 工程)、M (数学) 四门学科, 课程教学目 标分别从知识与技能、过程与方法、情感态度与价值观三个 维度来阐述, 具体内容如表 1 。

表 1 《暴躁的天气》STEM 教学目标

\begin{tabular}{|c|c|c|c|}
\hline STEM 目标 & 知识与技能 & 过程与方法 & 情感态度与价值观 \\
\hline $\mathrm{S}$ (科学) & $\begin{array}{l}\text { 认识各种灾害天气特征, 体会灾害天 } \\
\text { 气对人们生活的影响 }\end{array}$ & $\begin{array}{l}\text { 通过多种媒体感受各种灾害天气特征 } \\
\text { 及影响 }\end{array}$ & $\begin{array}{l}\text { 体验灾害天气给人们带来的 } \\
\text { 危害 }\end{array}$ \\
\hline T（技术） & $\begin{array}{l}\text { 学会使用天气测量工具来准确测量天 } \\
\text { 气数据 }\end{array}$ & $\begin{array}{l}\text { 通过信息检索、小组合作等方式掌握 } \\
\text { 使用天气测量工具 }\end{array}$ & 体验合作的精神 \\
\hline E (工程) & $\begin{array}{l}\text { 学会设计解决方案, 能够运用工程思 } \\
\text { 想设计合理的抵御灾害天气的建筑模 } \\
\text { 型 }\end{array}$ & $\begin{array}{l}\text { 掌握运用各种材料组装搭建模型的方 } \\
\text { 法 }\end{array}$ & $\begin{array}{l}\text { 在制作建筑模型过程中, 产 } \\
\text { 生对大自然的敬畏之心。 }\end{array}$ \\
\hline M (数学) & $\begin{array}{l}\text { 学会统计各种天气数据, 并且依据数 } \\
\text { 据推测天气状况 }\end{array}$ & 掌握简单的数学统计方法 & 培养严谨的科学态度 \\
\hline
\end{tabular}

\section{2 基于 STEM 理念的课程《暴躁的天气》教学 设计}

基于 STEM 理念的 5 E 探究式教学模式突出“探究”过程, 强调学习者敢于提出质疑并逐步激发探究思维，构建对新概 念的认知的过程。通过主题内容打通学科与学科的界限, 通 过不同的学科视域透视关于暴躁天气的问题，构成不同学科
能力要求的 STEM 教学设计, 即培养科学 (S) 的探究能力、 技术 $(\mathrm{T})$ 协作能力、数学 $(\mathrm{M})$ 的问题解决能力以及工程 $(\mathrm{E})$ 设计能力。

本研究结合基于 STEM 理念的 $5 \mathrm{E}$ 探究式教学模 式, 设计实施各种学习活动。具体的教学设计流程如图 1 所示。 


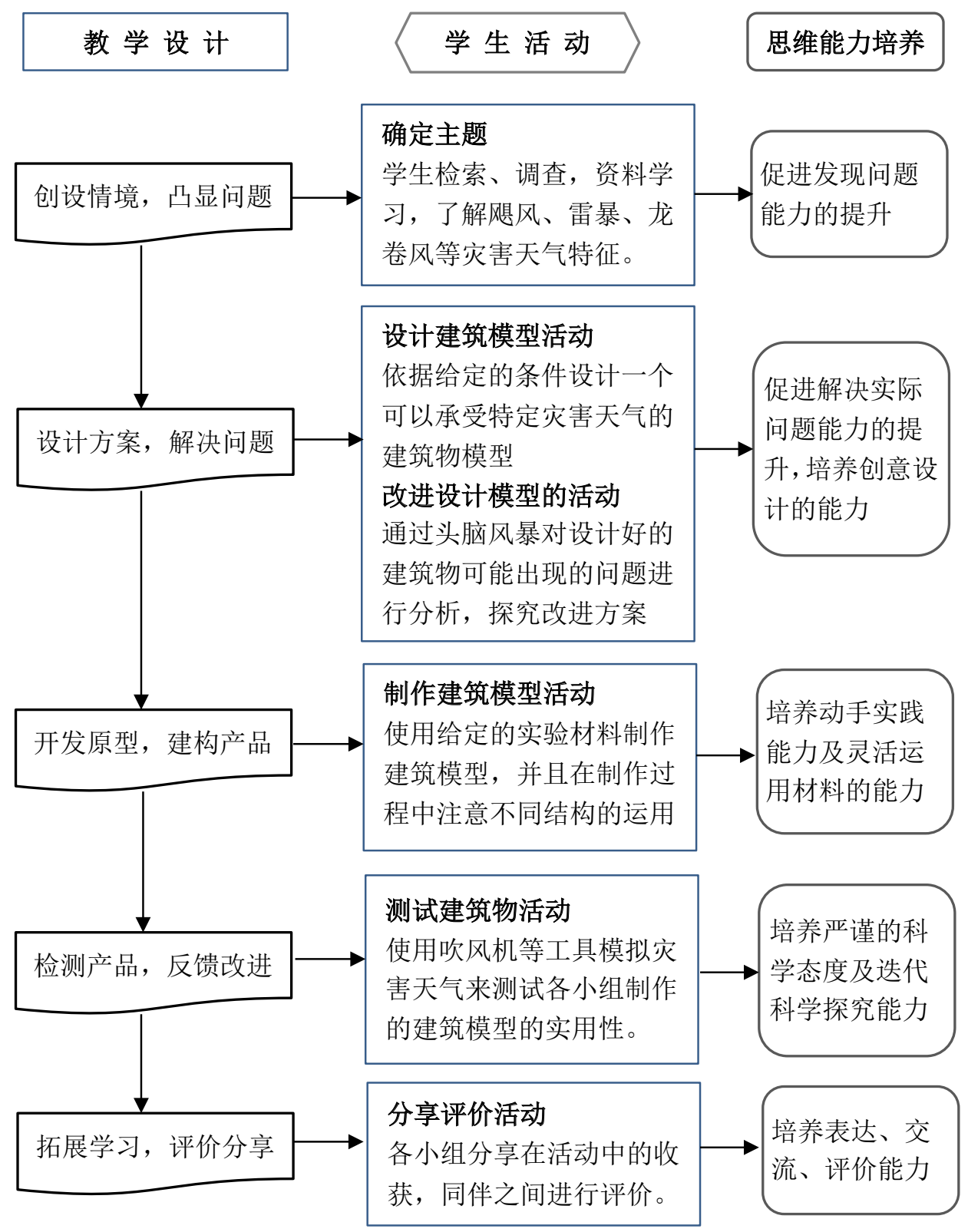

图 1 《暴躁的天气》教学设计与实施

该教学设计在首都师范大学附属中学大兴南校区小学 五年级进行了实施应用。实施过程根据学生在实际课堂中的 表现, 如对问题情境的理解程度、对活动的参与程度, 适时 进行调整。

\section{3 基于 STEM 理念的教学效果评测}

为了探究 STEM 理念下的跨学科教学对学生探究能力
的影响, 本研究采用单组前后测实验设计。以首都师范大学 附属中学大兴南校区小学五年级二班 38 名学生为研究对象, 以基于 STEM 的小学科学教学为自变量, 以学生科学探究 能力为因变量, 采用《STEM 教学成效调查问卷》为测量工具, 探讨基于 STEM 的跨学科教学对学生科学探究能力的影响。 研究从探究能力的八个维度, 分别对收集到的数据进行配对 样本 $\mathrm{T}$ 检验, 研究数据如表 2 所示。 
表 2 学习者科学探究能力前后测数据分析

\begin{tabular}{c|l|c|c|c|c|c}
\hline \multirow{2}{*}{ 维度 } & \multicolumn{1}{|c|}{ 题目 } & $\begin{array}{c}\text { 前测 } \\
\text { 均值 }\end{array}$ & $\begin{array}{c}\text { 前测 } \\
\text { 标准差 }\end{array}$ & $\begin{array}{c}\text { 后测 } \\
\text { 均值 }\end{array}$ & $\begin{array}{c}\text { 后测 } \\
\text { 标准差 }\end{array}$ & \multicolumn{1}{c}{$\mathrm{T}$ 值 } \\
\hline 提出问题 & 可以了解科学课上要解决的问题 & 4.121 & 0.823 & 4.334 & 0.733 & $-2.023 * *$ \\
\hline 作出假设 & 能够对科学课上观察的现象做出猜测与假设 & 4.116 & 0.879 & 4.349 & 0.720 & $-2.281 * *$ \\
\hline 制订计划 & 能够对自己发现的问题提出猜想方案, 并制定研究计划 & 4.209 & 0.940 & 4.419 & 0.794 & $-2.123 *$ \\
\hline \multirow{2}{*}{ 搜集证据 } & 能够将科学课上观察的现象记录下来 & 4.069 & 0.737 & 4.349 & 0.719 & $-1.296 * *$ \\
\cline { 2 - 6 } & 能够依据需要运用网络搜集相关的资料 & 4.213 & 1.035 & 4.256 & 0.727 & $-1.720 * *$ \\
\hline 处理信息 & 能够依据设计计划方案进行试验 & 3.977 & 0.727 & 4.256 & 0.734 & $-2.128 * *$ \\
\hline 得出结论 & 能够依据试验结果得出恰当的结论 & 3.628 & 1.254 & 4.232 & 0.922 & $-3.341 * *$ \\
\hline 表达交流 & 能够将自己的问题解决过程或结论讲解给同学听 & 3.837 & 1.090 & 4.186 & 0.982 & $-2.690 * *$ \\
\hline 反思评价 & $\begin{array}{l}\text { 能够及时反思在研究过程中的问题, 并且客观评价自己 } \\
\text { 及同伴的表现 }\end{array}$ & 3.628 & 1.254 & 4.232 & 0.922 & $-1.651 *$ \\
\hline
\end{tabular}

注: ${ }^{*} \mathrm{p}<0.05, * * \mathrm{p}<0.01$ 。

由表 2 可知, 经过此次 STEM 教学之后, 学生对于科 学探究能力了的七个维度的均值均达 4.0 以上, 表明学生的 科学探究能力有所提高。同时, 学生在提出问题、作出假设、 搜集证据、处理信息、得出结论、表达交流等几个方面的能 力较进行 STEM 教学之前有了显著性的变化 $(\mathrm{p}<0.01)$ 。可见, 以主题项目驱动展开的多学科融合的 STEM 教学, 学生更 有兴趣自主参与学习的全过程, 并且在学习过程中学生的科 学探究能力有了较大提高。即基于 STEM 的跨学科学习更 能促进小学生科学探究能力的提高。

另外, 研究数据表明学生在制定计划、反思评价等方 面的能力没有显著变化, 这可能与学生的学习习惯及逻辑思 维能力有关。学生的学习习惯及逻辑思维能力的培养是一个 过程, 不是通过一两次课程就能改善的。

\section{4 总结与思考}

本研究采用了基于 STEM 理念的 $5 \mathrm{E}$ 教学模式进行教学 设计, 并且以小学五年级《暴躁的天气》系列课程为例进 行了基于 STEM 理念的跨学科教学设计与实施。采用单组 前后侧准实验研究法, 从学生科学探究能力变化的视角, 对 STEM 跨学科教学对学生的影响进行了研究。通过对研究数 据及学生探究过程的分析发现, 基于 STEM 的跨学科教学 能够促进学生探究能力的提高。

由于本研究对 STEM 课程仍处于探索阶段, 教师不完
全具备 STEM 课程所需的综合素质以及条件, 而且本次实 验的设计只是单组实验, 缺少对照组, 问卷调查的数据并没 有达到预期的效果。由于教师个人能力的限制, STEM 跨学 科课程的教学设计还有待完善。在今后的研究中, 结合教学 模式, 还需结合主题开展更广泛的研究以及进行更科学的规 范, 继续完善 STEM 课程。

\section{参考文献}

[1] 中国教育科学研究院.中国 STEM 教育白皮书 (精华版) [EB/ OL].http://www.ict.edu.cn/uploadfile/2018/0507/20180507033914363. pdf,2017-06-20.

[2] 义务教育小学科学课程标准 [EB/OL].http://www.moe.gov.cn/srcsite/ A26/s8001/201702/W020170215542129302110.pdf,2017-02-15.

[3] Berry M R, Chalmers C, Chandra V. STEM futures and practice, can we teach STEM in a more meaningful and integrated way?[A]. Beijing Normal University and Griffith University. 2nd international STEM in education conference[C]. Beijing: Beijing Normal University, 2012:225-240.

[4] 余胜泉, 胡翔.STEM 教育理念与跨学科整合模式 [J]. 开放教育 研究 ,2015,(4):13-22.

[5] 傅寒, 刘鹏飞(2016). 从验证到创造——小学 STEM 教育应 用模式研究 [J]. 中国电化教育,(4):71-78.

[6] 李春密, 梁洁, 蔡美洁. 中学生科学探究能力结构模型初探 [J]. 课程教材教法, 2004, （6）：86-90

[7] 赵呈领, 赵文君, 蒋志辉. 面向 STEM 教育的 $5 \mathrm{E}$ 探究式教学 模式设计 [J]. 现代教育技术，2008 (3):106-112. 